\title{
CLINICOPATHOLOGIC CORRELATION OF CALRETININ EXPRESSION IN BREAST CANCER
}

\author{
Mayada S Farrag ${ }^{1}$, Amro A El-karef ${ }^{1}$, Maha M Amin ${ }^{1}$, Nagwa M Helal ${ }^{1}$ and Omar F Ali ${ }^{2}$ \\ ${ }^{1}$ Department of Pathology, Faculty of Medicine, Mansoura University, Mansoura, Egypt. \\ ${ }^{2}$ Department of Oncology Surgery, Faculty of Medicine, Mansoura University, Mansoura, Egypt.
}

\section{ABSTRACT}

Introduction and aims: It was found that calretinin is expressed in many tumors other than mesothelioma including breast carcinoma. We aim to assess calretinin expression in correlation with other clinicopathological parameters of breast carcinoma.

Methods: By using tissue microarrays, 225 breast cancer cases were immunostained for calretinin, estrogen receptors, progesterone receptors, and HER2.

Results: Tumors of high grade showed high calretinin expression $(\mathrm{P}<.0001)$. High loco-regional recurrence was also associated with high expression of calretinin $(\mathrm{P}=.005)$. Negative ER showed significant association with high calretinin expression $(\mathrm{P}=.005)$. Negative $\mathrm{PR}$, positive Her2neu showed also high significant association with high calretinin expression $(\mathrm{P}<.001)$.

Conclusion: Calretinin expression is high in high grade hormone negative tumors

Keywords: Calretinin, breast carcinoma.

Corresponding author:

Received: June 2016

Name: Mayada S Farrag

Tel. : 01061530778

Accepted: July 2016

\section{INTRODUCTION}

B reast cancer is the most frequently diagnosed cancer and the leading cause of cancer death among females worldwide ${ }^{[1]}$. In Egypt, it ranks the first and represents about $34 \%$ of female cancers according to the last registry of Egyptian National Cancer Institute [2].

Calretinin (CR) is a 29-kilodalton calcium-binding protein of the calmodulin superfamily. It can act as a buffer to guard against abnormal intracellular increase of calcium ${ }^{[3]}$. It was used as a diagnostic marker in the distinction between mesothelioma and lung adenocarcinoma. However, next studies showed that calretinin can also be expressed in several other cancers ${ }^{[3]}$.

Recently, Taliano et al. discovered highlevel of calretinin in high grade breast carcinoma particularly tumors with a basal-like phenotype. They also discovered that it is associated with poor overall survival. They suggested that it is a strong predictor of bad prognosis ${ }^{[4]}$.
In this study, we aimed at assessing calretinin expression in cases of invasive breast cancer in correlation with other clinicopathological parameters.

\section{MATERIALS AND METHODS}

Our study was a retrospective study carried out in surgical pathology lab at Oncology Center, Mansoura, Egypt on 225 formalin-fixed paraffin embedded mastectomy specimens of invasive breast carcinoma. We chose them randomly among cases who did not receive preoperative neoadjuvant chemotherapy or radiotherapy. We followed the guidelines of Mansoura university ethical committee (Code No. $\mathrm{PhD} / 22 / 2014)$.

Tissue microarrays were constructed manually. We revised all clinicopathological parameters of these 225 cases including age, location, size, multiplicity, histological type, and histological grade. Tumors were graded according to the Nottingham modification of the Bloom-Richardson scoring system [5]. Lymphovascular invasion, necrosis, metaplastic 
component, number of LN metastases $(\mathrm{N})$, distant metastasis $(\mathrm{M})$, and TNM staging were also assessed.

Sections from paraffin-embedded tissue microarrays were cut at $4 \mu \mathrm{m}$, deparaffinized with xylene, and rehydrated with graded alcohols. As regards antigen retrieval, it was done by heat in target retrieval solution $\mathrm{pH} 6.0$ for ER, PR, HER2, and calretinin. Antibodies for ER (rabbit monoclonal, clone SP1, cell marque, USA), PR (mouse monoclonal, clone PgR636, Dako, Glostrup, Denmark) and HER2/neu (mouse monoclonal, clone CB-11, cell marque, USA) were used.

ER and PR stains were considered positive if expression was observed in more than $1 \%$ of tumor nuclei [6]. For HER2 status, We considered tumors negative with a score of 0 , and +1 . Tumors were considered positive with a score of +3 when strong complete membranous staining was observed in at least $10 \%$ of tumor cells [4]. Cases of score $2+$ were excluded.

As regards calretinin, nuclear and/or cytoplasmic staining was considered positive Table (1): Clinicopathological features of all cases.
$[7,8]$. We considered both negative and weak staining as low- level expression of calretinin. Both moderate and strong staining were considered as high- level expression of calretinin [4].

\section{RESULTS}

Cases of this study was distributed as $198(88 \%)$ of invasive carcinoma of no special type (NST), 17 cases (7.6\%) of invasive lobular carcinoma, 10 cases $(4.4 \%)$ of other subtypes. They included 5 cases of mixed ductal and lobular carcinoma, 1case of carcinoma with neuroendocrine features, 1 case of mucinous carcinoma, 2 cases of papillary carcinoma, and 1 case of carcinoma with medullary features. As regards Tumor grade, grade 2 tumors were 174 cases $(77.3 \%)$, while grade 3 included 42 cases $(18.7 \%)$, and only 9 cases were grade 1 $(4 \%)$. As regards tumor stage, stage 3 tumors were 120 cases $(53.3 \%)$, while stage 2 included 97 cases $(43.1 \%)$, and 8 cases were stage 1 (3.6\%). We followed-up them with mean follow-up 35.5 months. Calretinin expression was high in 72 cases (32\%) and low in 153 cases $(68 \%$ ) (tables 1, 2, 3 \& figures 1,2$)$.

\begin{tabular}{llc}
\hline & Age $(\mathrm{y})$ & No. $(\%)$ \\
\hline$<60$ & $155(68.9 \%)$ \\
$\geq 60$ & $70(31.1 \%)$ \\
\hline 2. & Menopausal Status & \\
-Premenopausal & $75(33.3 \%)$ \\
-postmenopausal & $150(66.7 \%)$ \\
\hline 3. & Multicentericity & \\
-Negative & $218(96.8 \%)$ \\
- Positive & $7(3.1 \%)$ \\
\hline 4. & Tumor size & \\
-T1 & $17(7.6 \%)$ \\
- T2 & $128(56.9 \%)$ \\
-T3 & $74(32.9 \%)$ \\
-T4 & $6(2.7 \%)$ \\
\hline LN & \\
-N+ & $168(74.7 \%)$ \\
-N stage & \\
N0 & \\
N1 & $57(25.3 \%)$ \\
N2 & $59(26.2 \%)$ \\
N3 & $56(24.9 \%)$ \\
\hline
\end{tabular}


6. Initial pathological stage (pTNM)

-Stage 1

-Stage 2

$8(3.6 \%)$

-Stage 3

7. Locoregional recurrence

-Negative

$216(96 \%)$

-Positive

$9(4 \%)$

8. Metastasis

-Negative

$180(80 \%)$

-Positive

$45 \quad 20 \%)$

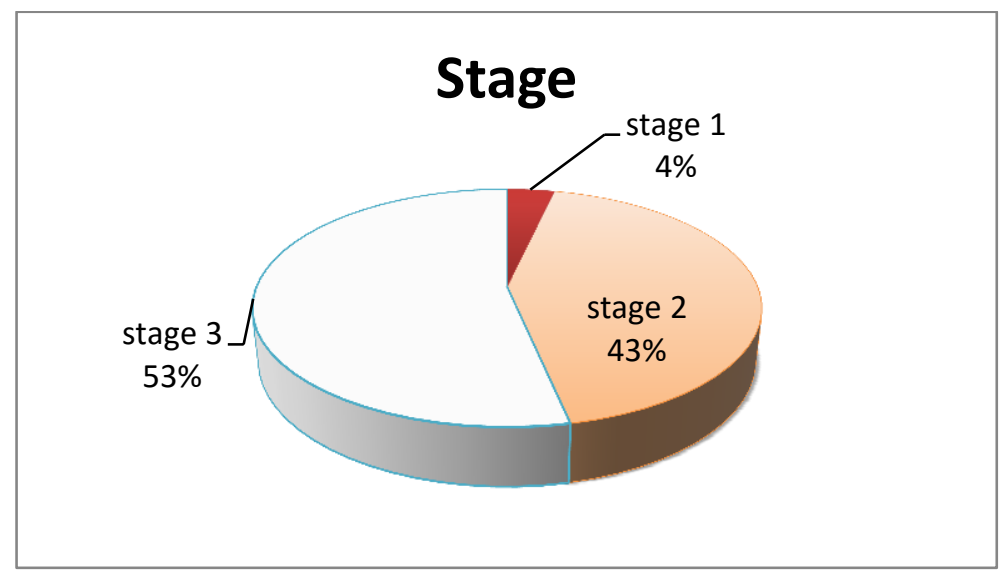

Figure (1): Staging of 225 cases of invasive breast carcinoma.

Table (2): Histologic features of all cases:

\begin{tabular}{ll}
\hline & \multicolumn{1}{c}{ No. $(\%)$} \\
\hline 1. Histologic Type & \\
- Invasive carcinoma of no special type (NST) & $198(88 \%)$ \\
- Invasive lobular carcinoma & $17(7.6 \%)$ \\
- Other subtypes & $10(4.4 \%)$ \\
\hline 2. Histologic grade & \\
- Grade1 & $9(4 \%)$ \\
- Grade2 & $174(77.3 \%)$ \\
- Grade3 & $42(18.7 \%)$ \\
\hline 3. Lymphovascular emboli & $178(79.1 \%)$ \\
-Negative & $47(20.9 \%)$ \\
- Positive & \\
4. Necrosis & $220(97.8 \%)$ \\
-Negative & $5(2.2 \%)$ \\
- Positive & $222(98.7 \%)$ \\
\hline 5. Squamous differentiation & $3(1.3 \%)$ \\
-Negative & \\
- Positive & \\
\hline
\end{tabular}




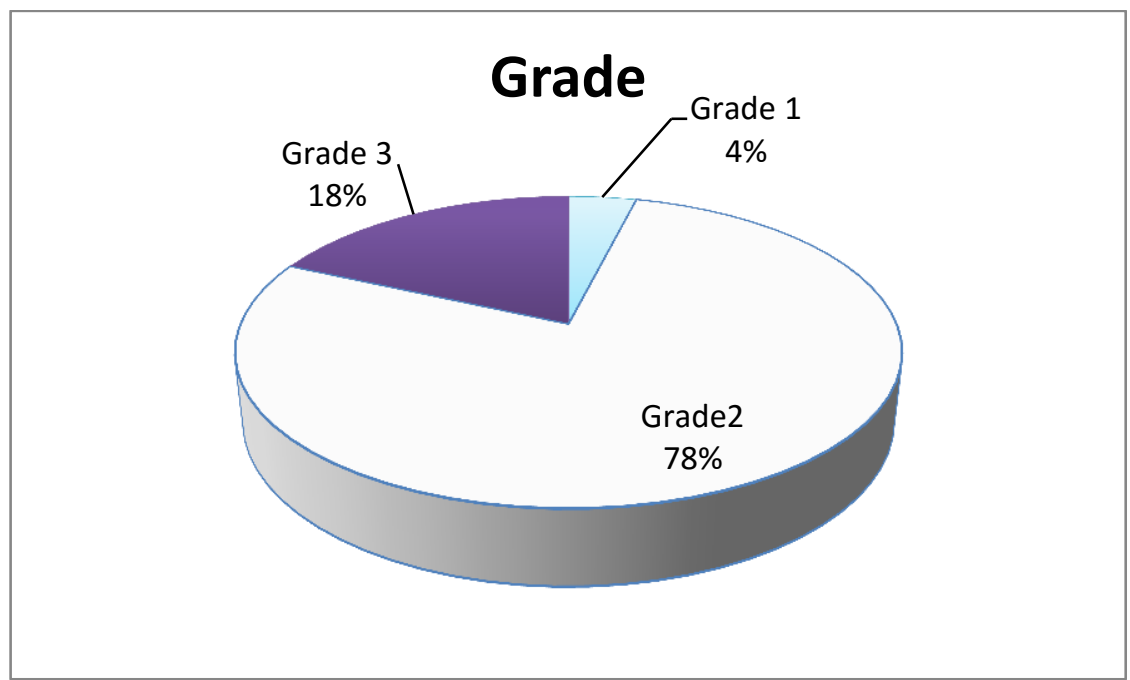

Figure (2): Grading of 225 cases of breast carcinoma.

Table (3): Immunohistochemical results of 225 cases:

\begin{tabular}{lll}
\hline & \multicolumn{1}{c}{ No. $(\%)$} \\
\hline 1. & Calretinin & \\
- & Low expression $(0,1)$ & $153(68 \%)$ \\
- & High expression $(2,3)$ & $72(32 \%)$ \\
\hline 2. & ER & \\
- & ER - & $126(56 \%)$ \\
- & ER+ & $99(44 \%)$ \\
\hline 3. & PR & \\
- & PR- & $111(49.3 \%)$ \\
- & PR+ & $114(50.7 \%)$ \\
\hline 4. & HER2 & \\
- & HER2- & $183(81.3 \%)$ \\
- & HER2+ & $42(18.7 \%)$ \\
\hline
\end{tabular}

Calretinin expression was high in 72 cases (32\%) and low in 153 cases (68\%) (figure 3). 


\section{Calretinin Expression}

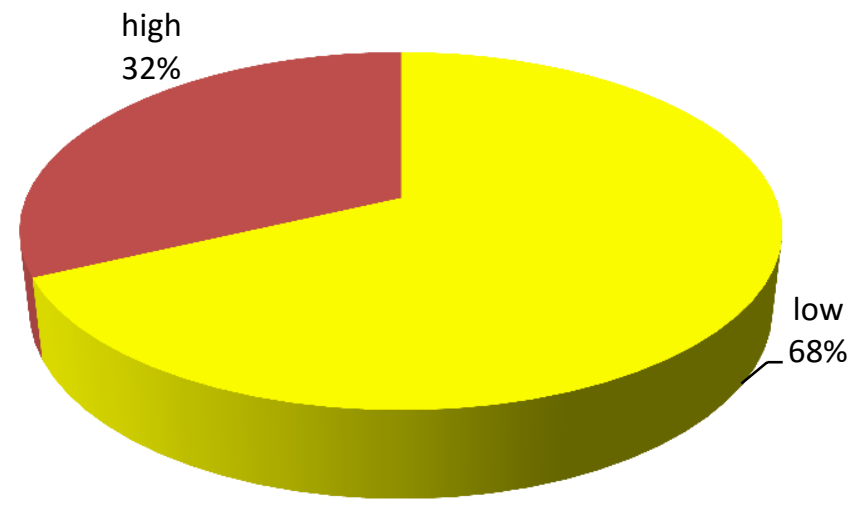

Figure (3): Calretinin expression of 225 cases of breast carcinoma.

Among other clinicopathological parameters, only tumors with locoregional recurrence showed significant high calretinin expression $(\mathrm{P}=.005)$ (figure 4). High calretinin expression was also associated with positive nodal status and advanced stage but without a statistical significance (figure $5 \& 6)$.

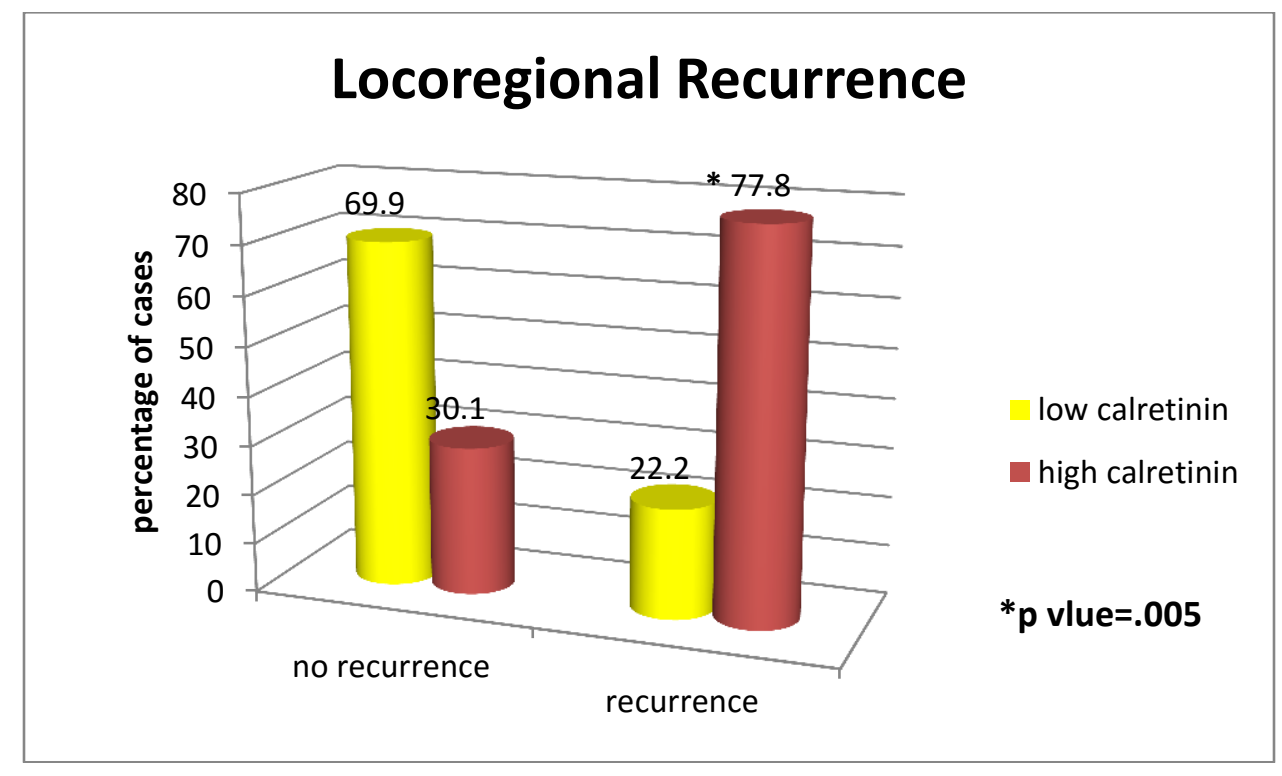

Figure (4): Locoregional recurrence in high and low calretinin expression cases. 


\section{Frequency of LN Metastases (N)}

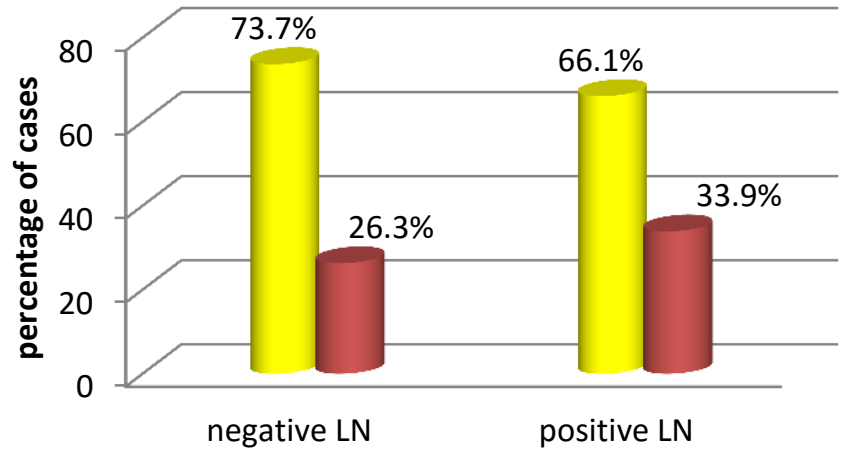

low calretinin

high calretinin

$p$ value $=.287$

Figure (5): Frequency of LN metastases in high and low calretinin expression cases.

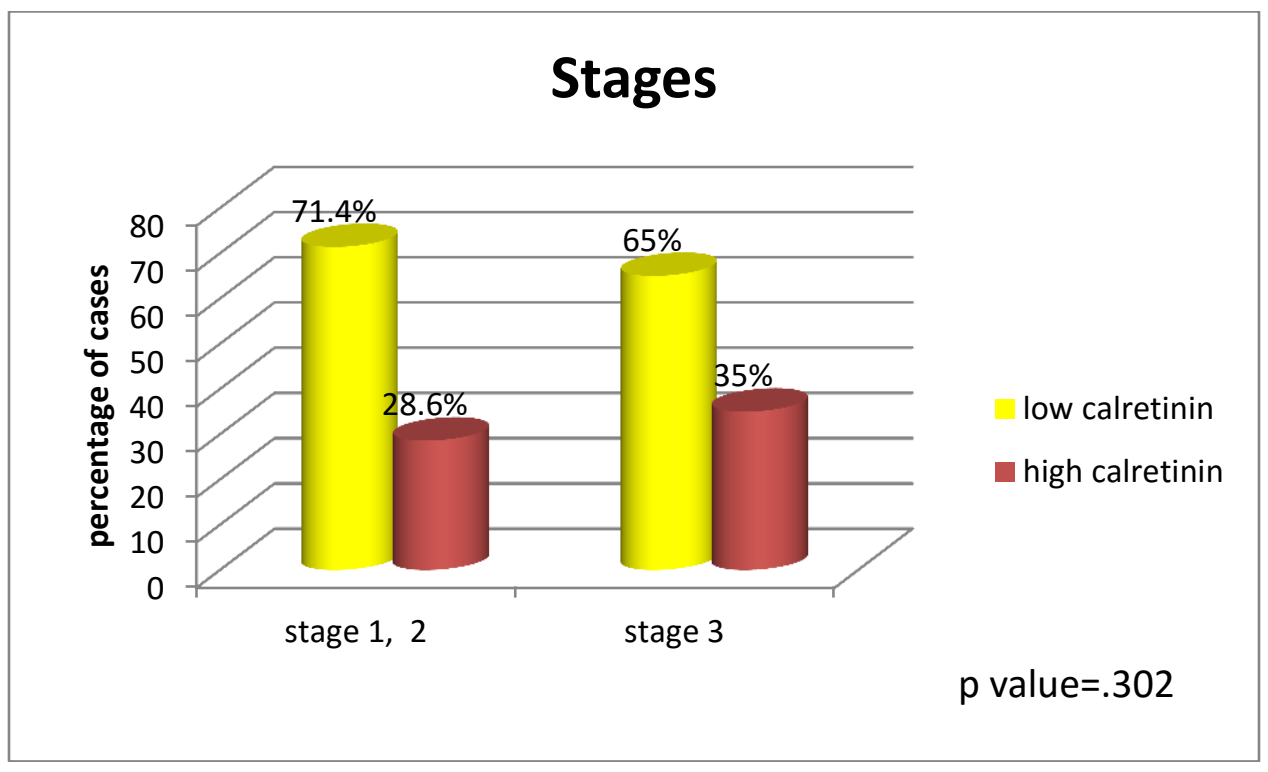

Figure (6): Staging in high and low calretinin expression cases.

Tumors with grade 3 showed significant high calretinin expression ( $\mathrm{P}<.001)$ (figure 7 ). As regards histologic type, invasive carcinoma of no special type (NST) showed significant high calretinin expression $(\mathrm{p}=.013)$. High calretinin expression was found only in $11.1 \%$ of other subtypes. No other histological parameters showed significant assossiation with calretinin expression. 


\section{Histological Grading}

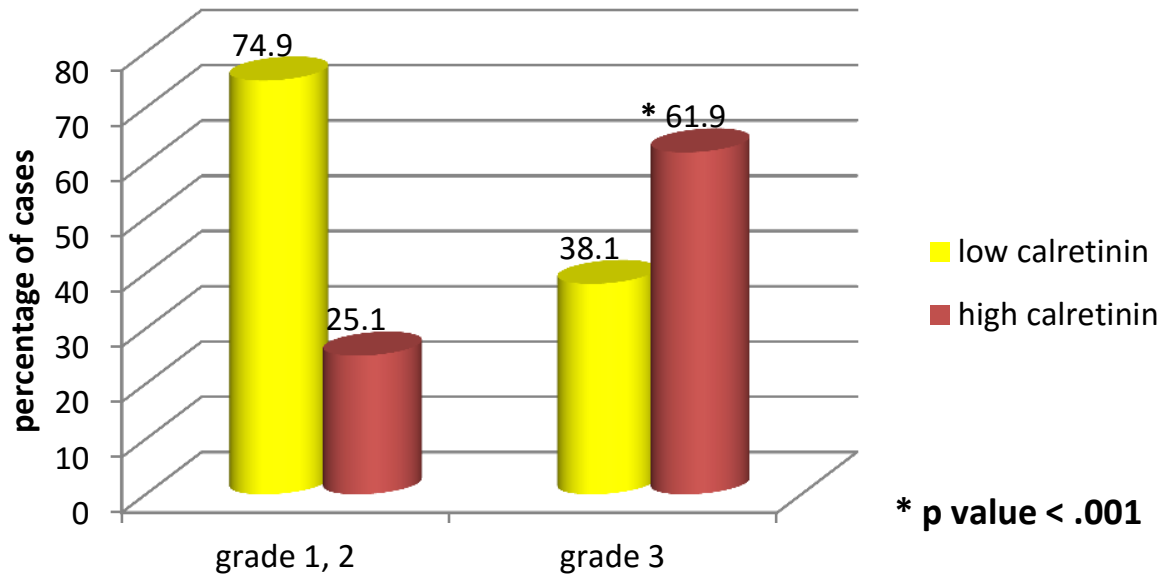

Figure (7): Histological grading in high and low calretinin expression cases.

Immunohistochemical stains revealed significant association between high calretinin expression and hormonal receptors (ER\&PR) and Her2 neu. Negative ER was significantly associated with high calretinin expression $(\mathrm{P}=$ .005). High calretinin expression was also significantly associated with negative PR, positive Her2neu $(\mathrm{P}<.001)$ (table 4$)$

Table (4): Associations of calretinin expression and other immunohistochemical markers:

\begin{tabular}{cccc}
$\begin{array}{c}\text { Calretinin Low } \\
\text { expression }(0,1)\end{array}$ & $\begin{array}{c}\text { Calretinin High } \\
\text { expression }(2,3)\end{array}$ & $\begin{array}{c}\text { Chi-square } \\
(\boldsymbol{\chi} \mathbf{2})\end{array}$ & P value \\
\cline { 1 - 2 }$=153)$ & $(\mathrm{N}=72)$ & & \\
\hline No. $(\%)$ & No. $(\%)$ &
\end{tabular}

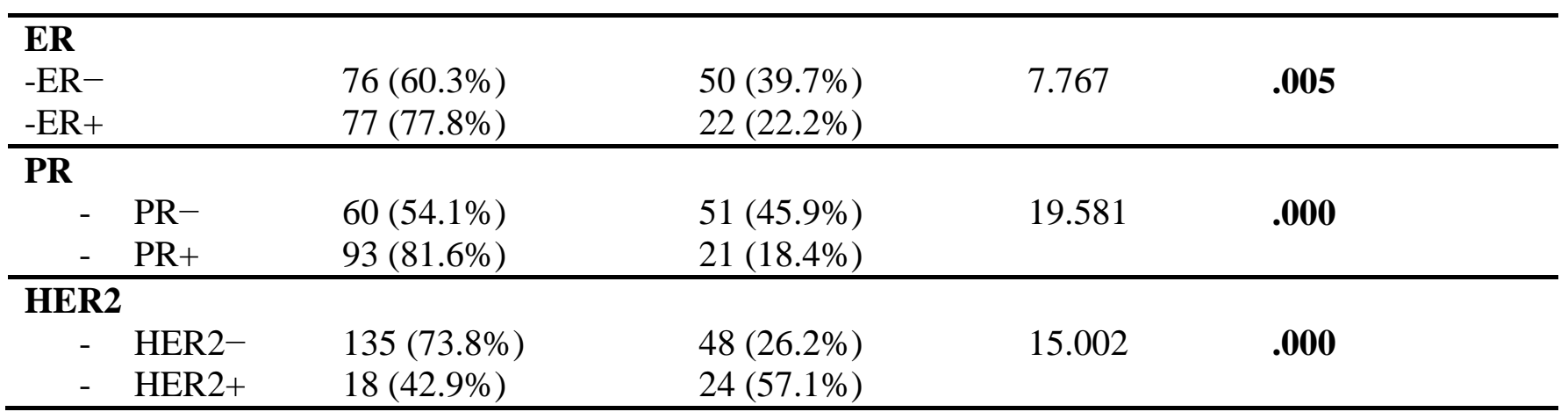

\section{DISCUSSION}

In this study, we assessed calretinin immunohistochemical staining in invasive breast cancer in correlation with other clinicopathological data. Only few previous studies discussed calretinin expression in breast.
In our study, we demonstrated calretinin expression in invasive cancer breast. It was found to be high in 72 cases $(32 \%)$ and low in 153 cases $(68 \%)$. Tumors with high histologic grade showed significant high calretinin expression if compared with lower grades 1, 2 . This result is in agreement with previous two 
studies done by Duhig et al., $\left.{ }^{[9}\right]$ and Taliano et al., ${ }^{[4]}$.

Taliano et al. ${ }^{[4]}$ showed that calretinin is strongly expressed in patients with highgrade (grade 3) invasive ductal carcinoma. Also, Duhig et al., [9] found immunohistochemical positivity in a significant proportion of high grade breast carcinomas.

Our study showed that loco-regional recurrence is statistically associated with high calretinin expression. No previous studies discussed this point. Histologic types of breast cancer differ in calretinin expression. Invasive carcinoma of no special type (NST) showed higher calretinin expression where 69 cases $(34.8 \%)$ of NST carcinoma cases showed that. On the other hand, only 3 cases $(11.1 \%)$ of other subtypes which showed high calretinin expression $\quad(\mathrm{P}=\quad .013)$. NO other clinicopathological and histological parameters were associated with significant calretinin expression.

In our study, we found a high statistically significant association between higher calretinin expression and adverse hormonal receptors status (negative ER and PR), positive Her2 neu. These findings were in agreement with previous other studies done by Powell et al. ${ }^{[10]}$, Duhig et al. [9] and Taliano et al., ${ }^{[4]}$.

Powell et al. [10] found statistically significant association between tumor calretinin expression and negative ER status. Only one of the eight (13\%) calretinin-positive tumours was also positive for ER, but 39 of the $45(87 \%)$ calretinin-negative tumours were positive for ER $(\mathrm{P}=0.000079)$.

Duhig et al. [9] also found an association between tumor calretinin expression and negative ER status. They found that 23 of the 38 ER-negative tumours (60.5\%) were positive for calretinin, but 5 of the $15(33.3 \%)$ ER-positive tumours were positive for calretinin.

Taliano et al., [4] found the same association between high expression of calretinin and negative hormonal status. They concluded that high calretinin expression in
$47.6 \%$ of ER negative tumors. On the other hand, only $8.9 \%$ of ER positive tumors showed high calretinin expression. Also, high calretinin expression was found in $41.3 \%$ of PR negative tumors. They found high calretinin expression only $13.2 \%$ of PR positive tumors $(\mathrm{P}<.001)$.

In this study, high calretinin expression showed also high statistically significant association with positive Her2neu where 24 cases of 42 cases of Her2 positive tumors (57.1\%) showed high calretinin expression versus 48 cases of 183 cases of Her2 negative tumors $(26.2 \%)$ which showed high calretinin expression $(\mathrm{P}<.001)$.

Duhig et al. [9], found positive calretinin expression in $40 \%$ of cases of positive Her2neu while Taliano et al., [4] found high calretinin expression in only $16.4 \%$ of cases of positive Her2neu.

\section{List of abbreviations}

CR Calretinin, ER Estrogen Receptor, Her2 Human epidermal growth factor receptor, IHC Immunohistochemistry, NST Invasive carcinoma of no special type.

\section{REFERENCES}

1. Jaemal, A., Bray, F., Center, M. M., Ferlay, J., Ward, E., \& Forman, D. (2011). Global cancer statistics. CA: a cancer journal for clinicians, 61(2), 69-90.

2. Mokhtar, N., Gouda, I., \& Adel, I. (2007). Cancer pathology registry 2003-2004 and time trend analysis. Department of pathology, NCI.

3. Lugli, A., Forster, Y., Haas, P., Nocito, A., Bucher, C., Bissig, H., \& Sauter, G. (2003). Calretinin expression in human normal and neoplastic tissues: a tissue microarray analysis on 5233 tissue samples. Human pathology, 34(10), 994-1000.

4. Taliano, R. J., Lu, S., Singh, K., Mangray, S., Tavares, R., Noble, L., \& Yakirevich, E. (2013). Calretinin expression in highgrade invasive ductal carcinoma of the breast is associated with basal-like subtype and unfavorable prognosis. Human pathology, 44(12), 2743-2750.

5. Elston, C. W., \& Ellis, I. O. (1991). Pathological prognostic factors in breast 
cancer. I. The value of histological grade in breast cancer: experience from a large study with long-term followup. Histopathology, 19(5), 403-410.

6. Goldhirsch, A. 2., Wood, W. C., Coates, A. S., Gelber, R. D., Thürlimann, B., \& Senn, H. J. (2011). Strategies for subtypesdealing with the diversity of breast cancer: highlights of the St Gallen International Expert Consensus on the Primary Therapy of Early Breast Cancer 2011. Annals of oncology, mdr304.

7. Fernandez-Flores, A. (2013). Cutaneous metastases from breast carcinoma: calretinin expression and estrogen, progesterone and Her2/neu status of the metastases, compared to primary cutaneous apocrine tumors. Romanian journal of morphology and embryology= Revue roumaine de morphologie et embryologie, 54(3 Suppl)

8. Altaf, F. J., Mokhtar, G. A., Emam, E., Bokhary, R. Y., Mahfouz, N. B., Al Amoudi, S., \& AL-Gaithy, Z. K. (2014). Metaplastic carcinoma of the breast: an immunohistochemical study. Diagn Pathol, 9, 139.

9. Duhig, E. E., Kalpakos, L., Yang, I. A., \& Clarke, B. E. (2011). Mesothelial markers in high-grade breast carcinoma. Histopathology, 59(5), $\quad 957-$ 964.

10. Powell, G., Roche, H., \& Roche, W. R. (2011). Expression of calretinin by breast carcinoma and the potential for misdiagnosis of mesothelioma.Histopathology, 59(5), 950956. 